\title{
Performance measurements of mode-converting corrugated horns
}

\author{
Craig R. Donaldson, Paul McElhinney, Liang Zhang, Adrian W. Cross, and Wenlong He \\ Department of Physics, SUPA, University of Strathclyde \\ Glasgow, Scotland, UK, G4 ONG \\ liang.zhang@strath.ac.uk
}

\begin{abstract}
This paper presents the design, simulation and measurement of two mode converting corrugated horns for application as the output launcher for a gyro-amplifier. Operating over the 90 to $100 \mathrm{GHz}$ frequency range the horns act to convert the $\mathrm{TE}_{11}$ mode to the Gaussian-like $\mathrm{HE}_{11}$ mode. The difference in the horns is one was made initially to meet a more moderate Gaussian content specification while the other was improved to meet stricter criteria on Guassian content as-well-as being vacuum tight and compatible with integration with a broadband multi-layer vacuum window. The results are presented and show that in both cases the measured results agree well with simulated values and ultimately the second horn was demonstrated to be a good candidate to be tested on a gyrotron amplifier.
\end{abstract}

\section{INTRODUCTION}

A high power gyrotron-traveling wave amplifier (gyroTWA) operating in the W-band frequency range has been developed at the University of Strathclyde. Such amplifiers are currently in demand due to their many promising applications [1]. The gyro-TWA uses a helically corrugated interaction region [2,3] and a cusp electron gun [4] that generates a $40 \mathrm{kV}, 1.5 \mathrm{~A}$ axis-encircling electron beam. It was numerically simulated to generate $\sim 5 \mathrm{~kW}$ microwave power over the $90 \mathrm{GHz}$ to $100 \mathrm{GHz}$ frequency range. For many applications it is beneficial for the microwave beam to be in the form of a fundamental Gaussian mode and therefore a mode converter is required after the interaction region which outputs the circularly polarised $\mathrm{TE}_{11}$ mode. It is usual to do this through the output horn which can be either corrugated or smoothly profiled [5]. In this paper two corrugated horns based on a $\sin ^{2}$ profile were considered. First, a prototype horn was designed to verify the design principle and a second improved design integrated with a multilayer window, hereafter called mark 1 [6] and mark 2 [7] respectively, are presented with the measured microwave results discussed.

\section{CORRUGATED HORN MARK 1 AND 2}

The microwave properties of a corrugated horn were calculated through the mode-matching method [8]. This is especially useful due to the numerous number of corrugations as-well-as the relatively wide and long structure compared to the operating wavelength.
Initially a corrugation profile that followed a $\sin ^{2}$ line, as described by Clarricoats and Olver [9] was used as the baseline and then the structure was optimised around this. It was found that although other profiles exist this one had a good balance of mode purity and directivity while keeping reflections low, better than $-30 \mathrm{~dB}$ over the wide frequency range. The geometry of the horn was also constrained by the existing dimensions of the gyro-TWA. The input diameter was fixed by the output of the interaction circuit while the horn profile had to be take into consideration the electron beam trajectories so as to not intercept them, since a beam dump will be added at a future date. The numerical optimisation was performed with the goal of obtaining the previously discussed mode mixture of the $\mathrm{TE}_{11}$ and $\mathrm{TM}_{11}$ modes as well as keeping the reflection lower than $-30 \mathrm{~dB}$.

The mark 1 design, as shown in Fig. 1, had a fixed input diameter of $5.6 \mathrm{~mm}$. After the corrugated horn was optimised it was found that the ideal profile had 101 corrugations, with a depth varying from $\lambda / 2$ to $\lambda / 4$, and was simulated to have lower than $-35 \mathrm{~dB}$ reflection with Gaussian coupling efficiency of $\sim 98 \%$.

The mark 2 design, as shown in Fig. 1, had a requirement to improve the Gaussian coupling efficiency to over $99 \%$ with an integrated UHV vacuum tight multilayer window. It was decided to reduce the input diameter to exactly match the output of the HCW at $2.9 \mathrm{~mm}$ and in order to increase the coupling efficiency an additional phase matching section after the horn profile was used. The simulation showed over $99 \%$ Gaussian content with reflection of below $-30 \mathrm{~dB}$.

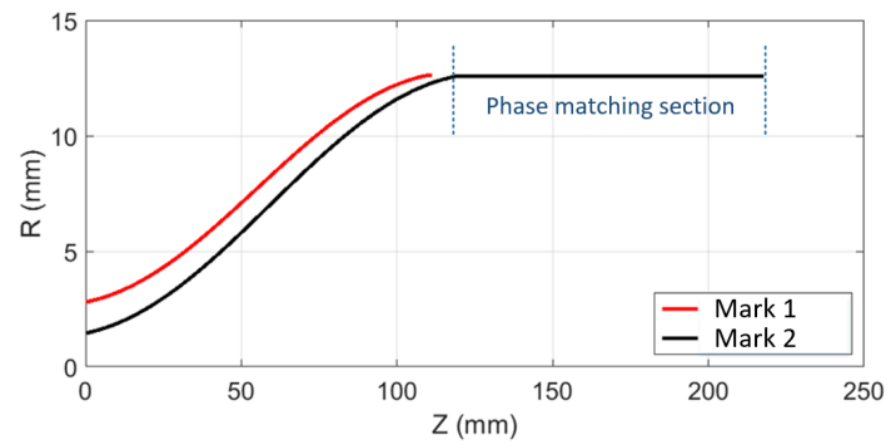

Fig 1. The corrugation profile for both mark 1 and mark 2 horns. 


\section{MEASURED RESULTS}

Both horns were constructed through the electroforming method. Mark 1 was made as a simple solid horn while mark 2 had two CF flanges grown into it. On the input end was a CF35 flange and at the output there was a CF70 flange. A multilayer window was also connected to the output flange of the horn, details of which can be found in [10].

An Anritsu MEXXXX vector network analyser was used to measure the microwave properties of each horn including reflection, far-field and near-field patterns. Mark 1 showed a reflection of less than $-30 \mathrm{~dB}$ while the far-field patterns, shown in Fig 2. agreed very well with numerical simulations. It was therefore determined that the horn had a Gaussian coupling efficiency of $\sim 98 \%$ with a directivity of $26.6 \mathrm{GHz}$ at the centre frequency of $95 \mathrm{GHz}$.

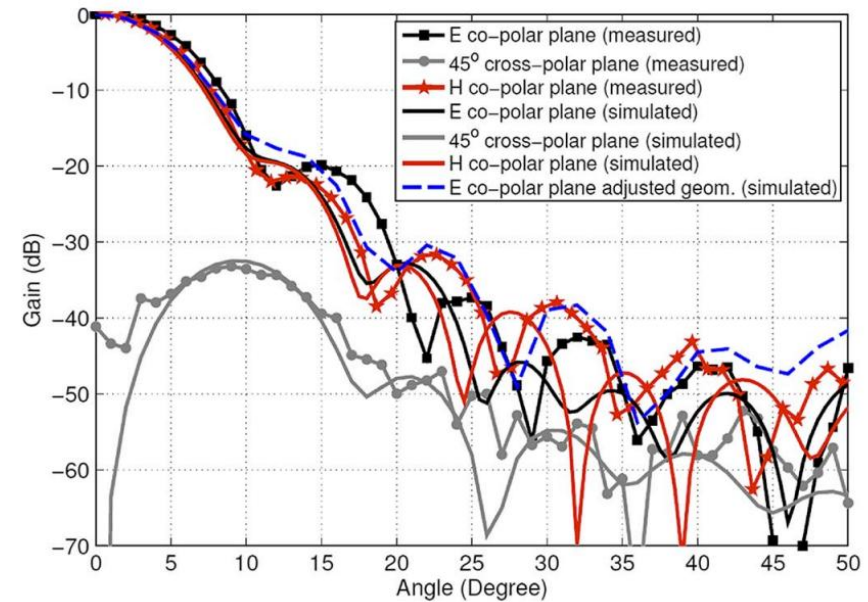

Fig 2. Measured and simulated far-field patterns for corrugated horn "mark 1".

The mark 2 horn was made and combined with the multilayer window. This was vacuum leak checked achieving a measured vacuum pressure of $1 \mathrm{e}-9 \mathrm{mBar}$. The horn was then measured with and without the multilayer window attached, as the addition of the window will add some reflections. The reflection showed a measured return loss of between -30 and $40 \mathrm{~dB}$ over the 90 to $100 \mathrm{GHz}$ frequency range. The addition of the window did not effect the reflection measurement significantly which shows the window has very low reflection itself. The measured far-field patterns agreed very well to the simulations.

\section{CONCLUSION}

In conclusion two corrugated horns were designed, simulated and measured. One was a prototype with a $\sin ^{2}$ profile while the other had an additional phase matching section while being combined with a multilayer window. Each horn was simulated and optimised before being made and measured on a VNA. The measured result in both cases showed excellent agreement with simulations and the mark 2 horn was demonstrated to be vacuum tight and compatible for use with a W-band gyroTWA.

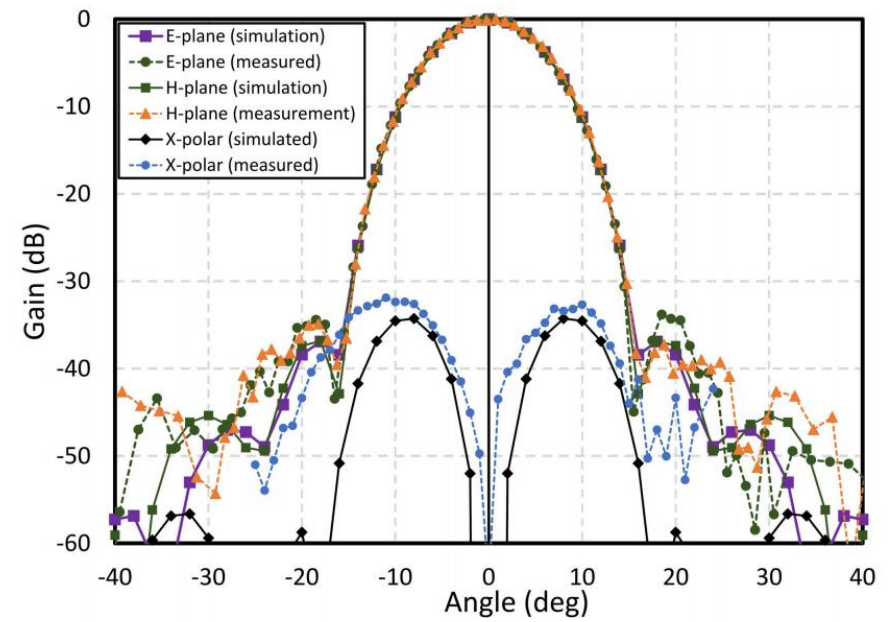

Fig 3. Measured and simulated far-field patterns for the corrugated horn "mark 2 ".

\section{REFERENCES}

[1] W. He, et al., "High power wideband gyrotron backward wave oscillator operating towards the terahertz region," Phys. Rev. Lett., 110, 165101, 2013

[2] L. Zhang, W. He, K. Ronald, A.D.R. Phelps, C.G. Whyte, C.W Robertson, A.R. Young, C.R. Donaldson, and A.W. Cross, "Multi-mode coupling wave theory for helically corrugated waveguide," IEEE Trans. Microw. Theory Techn., 60, pp. 1-6, 2012.

[3] A.W. Cross, W. He, A.D.R. Phelps, K. Ronald, C.G. Whyte, A.R Young, C.W. Robertson, E.G. Rafferty, and J. Thomson, "Helically corrugated waveguide gyrotron traveling wave amplifier using a thermionic cathode electron gun," Appl. Phys. Lett., 90, 253501, 2007.

[4] C.R. Donaldson, et al., "A cusp electron gun for millimeter wave gyrodevices," Appl. Phy. Lett., 96, 141501, 2010.

[5] L. Zhang, W. He, C.R. Donaldson, G.M. Smith, D.A. Robertson, R.I Hunter, and A.W. Cross, "Optimization and measurement of a smoothly profiled horn for a W-band gyro-TWA," IEEE Trans. Electron Dev., 64, pp. 2665-2669, 2017.

[6] P. McElhinney, C. R. Donaldson, L. Zhang, and W. He, "A high directivity broadband corrugated horn for W-band gyro-devices," IEEE Trans. Antennas Propag., 61, pp. 1453-1456, 2013.

[7] P. McElhinney, et al., "An output coupler for a W-band high powe wideband gyro-amplifier," IEEE Trans. Electron Devices, 64, pp. 1763 1766, 2017.

[8] Mician GmbH. (2012). $\mu$-Wave Wizard. [Online]. Available: http://www.mician.com

[9] P. J. B. Clarricoats and A. D. Olver, Corrugated Horns for Microwave Antennas. London, U.K.: Peregrinus, 1984

[10] C.R. Donaldson, P. McElhinney, L. Zhang, and W. He, "Wide-band $\mathrm{HE}_{11}$ mode terahertz wave windows gor gyro-amplifiers," IEEE Trans. THz Sci. Technol., 6, pp. 108-111, 2017. 